\title{
Heart valve flow computation with the integrated Space-Time VMS, Slip Interface, Topology Change and Isogeometric Discretization methods
}

\author{
Kenji Takizawa ${ }^{\mathrm{a}, *}$, Tayfun E. Tezduyar ${ }^{\mathrm{b}}$, Takuya Terahara $^{\mathrm{a}}$, Takafumi Sasaki ${ }^{\mathrm{a}}$ \\ ${ }^{a}$ Department of Modern Mechanical Engineering, Waseda University \\ 1-6-1 Nishi-Waseda, Shinjuku-ku, Tokyo 169-8050, Japan \\ ${ }^{b}$ Mechanical Engineering, Rice University - MS 321 \\ 6100 Main Street, Houston, TX 77005, USA
}

\begin{abstract}
Heart valve flow computation requires accurate representation of boundary layers near moving solid surfaces, including the valve leaflet surfaces, even when the leaflets come into contact. It also requires dealing with a high level of geometric complexity. We address these computational challenges with a space-time (ST) method developed by integrating three special ST methods in the framework of the ST Variational Multiscale (ST-VMS) method. The special methods are the ST Slip Interface (ST-SI) and ST Topology Change (ST-TC) methods and ST Isogeometric Analysis (ST-IGA). The computations are for a realistic aortic-valve model with prescribed valve leaflet motion and actual contact between the leaflets. The ST-VMS method functions as a moving-mesh method, which maintains high-resolution boundary layer representation near the solid surfaces, including leaflet surfaces. The ST-TC method was introduced for moving-mesh computation of flow problems with TC, such as contact between the leaflets of a heart valve. It deals with the contact while maintaining high-resolution representation near the leaflet surfaces. The ST-SI method was originally introduced to have high-resolution representation of the boundary layers near spinning solid surfaces. The mesh covering a spinning solid surface
\end{abstract}

\footnotetext{
${ }^{*}$ Corresponding author. Tel.: +81-3-5286-2143.

Email addresses: Kenji.Takizawa@tafsm.org (Kenji Takizawa), tezduyar@tafsm.org (Tayfun E. Tezduyar)
}

Preprint submitted to Computers 8 Fluids

November 11, 2016 
spins with it, and the SI between the spinning mesh and the rest of the mesh accurately connects the two sides. In the context of heart valves, the SI connects the sectors of meshes containing the leaflets, enabling a more effective mesh moving. In that context, integration of the ST-SI and ST-TC methods enables high-resolution representation even when the contact is between leaflets that are covered by meshes with SI. It also enables dealing with contact location change or contact and sliding on the SI. By integrating the ST-IGA with the ST-SI and ST-TC methods, in addition to having a more accurate representation of the surfaces and increased accuracy in the flow solution, the element density in the narrow spaces near the contact areas is kept at a reasonable level. Furthermore, because the flow representation in the contact area has a wider support in IGA, the flow computation method becomes more robust. The computations we present for an aortic-valve model with two different modes of prescribed leaflet motion show the effectiveness of the ST-SI-TC-IGA method.

Keywords: Heart valve flow, Contact, Space-Time VMS method, Space-Time Slip Interface method, Space-Time Topology Change method, Isogeometric Analysis, Mesh generation

\section{Introduction}

Heart valve flow computation requires accurate representation of boundary layers near moving solid surfaces, including the valve leaflet surfaces, even when the leaflets come into contact. At the same time it requires dealing with a high level of geometric complexity. We have addressed these computational challenges in a recent article $\langle 1>$, and this is an expanded, journal version of that.

In computation of flows with moving boundaries and interfaces (MBI), including heart valve flow, accurate representation of boundary layers near moving solid surfaces requires moving-mesh (interface-tracking) methods. In a movingmesh method, the fluid mechanics mesh follows (tracks) the fluid-solid interfaces, enabling us to control the mesh resolution near the interface and have 
high-resolution representation of the boundary layers.

The Space-Time Variational Multiscale (ST-VMS) method $\langle 2$; $3>$, the core method we are using here in addressing the challenges of heart valve flow computation, is a moving-mesh method. It was introduced mainly for flows with MBI, including fluid-structure interactions (FSI). It is the VMS version of the Deforming-Spatial-Domain/Stabilized ST (DSD/SST) method $\langle 4$; 5; 6 $>$, with the VMS components coming from the residual-based VMS (RBVMS) method $\langle 7$; , 8; 9; 10 $>$. The stabilization components of the original DSD/SST method are the Streamline-Upwind/Petrov-Galerkin (SUPG) $<11>$ and Pressure-Stabilizing/Petrov-Galerkin (PSPG) $<4>$ stabilizations, and for that the method is also called "ST-SUPS." Functionally, the ST-VMS method can be seen as an augmented version of the ST-SUPS method, with two additional stabilization terms beyond the three of the ST-SUPS method, or the ST-SUPS method can be seen as a reduced version of the ST-VMS method.

The ALE-VMS method $<12$; 13, 14 $>$ is the VMS version of the Arbitrary Lagrangian-Eulerian (ALE) finite element method $\langle\underline{15}\rangle$, which is a more commonly used moving-mesh method. The RBVMS and ALE-VMS methods have been successfully used for different types of FSI, MBI and fluid mechanics problems (see, for example, $<16$; 17, 12; 18, 19, 20, 21, 22, 14, 23, 24, 25, 26, 27, 28 , 29; 30; 31, 32; 33, 34; 35, 36, 37, 38, 39, 40>).

The ST-SUPS and ST-VMS methods have also been successfully used for different classes of FSI, MBI and fluid mechanics problems. The classes of problems include spacecraft parachute FSI $<25$, 41; 42, 43, 44, 45 $>$, windturbine aerodynamics $\langle 21$, 46, 47, 25, 48, 41, 49, 29, 50>, flapping-wing aerodynamics $\langle 51,52,53,25,54,55,56,41,49,57>$, cardiovascular fluid mechanics $\langle 58,59,55,60,41,49,61 ; 62>$, spacecraft aerodynamics $\langle 63,64>$, thermofluid analysis of ground vehicles and their tires $\langle 65\rangle$, thermo-fluid analysis of disk brakes $\langle 666>$, flow-driven string dynamics in turbomachinery $\langle 67>$, flow analysis of the turbine part of a turbocharger $\langle\underline{68}>$, flow around a tire with road contact and deformation $\langle\underline{69}>$, and ram-air parachutes $\langle\underline{70}>$.

The challenges associated with using a moving-mesh method in a heart valve 
flow computation even when the valve leaflets come into contact and create a topology change (TC) in the fluid mechanics domain is addressed with the STTC method $<\underline{55}>$. Even before the ST-TC method, the ST-SUPS and ST-VMS methods, when used with robust mesh update methods, have been proven effective in flow computations where the solid surfaces are in near contact or create other near TC, if the nearness is sufficiently near for the purpose of solving the problem. Many classes of problems can be solved that way with sufficient practical accuracy. For examples of such computations, see the references mentioned in $\langle 55\rangle$. Heart valve flow computation, however, is an example of the classes of computations where the nearness that can be modeled with a moving-mesh method without actually bringing the surfaces into contact might not be near enough. That is because an actual contact is required for blocking the flow when the valve closes. The Fluid-Solid Interface-Tracking/Interface-Capturing Technique (FSITICT) $<71>$ was motivated by that class of problems. In the FSITICT, we track the interfaces wherever and whenever we can with a moving mesh, and capture over that moving mesh the interfaces we cannot track, specifically the interfaces where and when we need to have an actual contact between the solid surfaces. As commented in $<62>$, essentially, the FSITICT is based on giving up on the interface-tracking accuracy where and when we expect an actual contact. The FSITICT has been successfully extended in $\langle 34>$ to 3D FSI computation of a bioprosthetic heart valve. In that case the interface-tracking technique was the ALE-VMS method, and the interface-capturing technique was a variational immersed-boundary method, later referred to as the Immersogeometric method in $\langle 72>$.

The ST-TC method does not give up on interface-tracking (moving-mesh) accuracy even when there is an actual contact between solid surfaces or other TC. It can handle an actual TC while maintaining high-resolution boundary layer representation near solid surfaces. The ST-TC method is based on special mesh generation and update, and a master-slave system that maintains the connectivity of the "parent" mesh when there is a TC. The ST-TC method was demonstrated in $2 \mathrm{D}$ test computations $<55>$ and has been successfully applied 
to $3 \mathrm{D}$ computational analysis of heart valve models $\langle 62>$ and wing clapping $<57>$.

Heart valve flow computations involve a high level of geometric complexity. Therefore it is desirable to have a good level of freedom in mesh generation and mesh moving. We accomplish that with the ST Slip Interface (ST-SI) method $<50>$. The method was originally introduced to retain the desirable movingmesh features of the ST-VMS method when we have spinning solid surfaces, such as a wind-turbine rotor. With the ST-SI method, the mesh covering the spinning solid surface spins with it and we maintain the high-resolution representation of the boundary layers. Earlier methods to accomplish that objective in the ST framework were the Shear-Slip Mesh Update Method (SSMUM) $\langle 73$; 74; 75>, which was introduced in $\langle 73 ;$ 74 $>$ and named "SSMUM" in $\langle 75\rangle$, and the ST/NURBS Mesh Update Method (STNMUM), which was introduced in $<51$, 52; 53> and named "STNMUM" in $<48>$. In the ST-SI method NURBS basis functions can be used for the temporal representation of the spinning motion. With the spinning motion represented by quadratic temporal NURBS basis functions, and with sufficient number of temporal patches for a full rotation, the circular paths associated with the spinning motion can be represented exactly. A secondary mapping $<2,51,3,25>$ enables also specifying a constant angular velocity corresponding to the invariant speeds along those paths. For more on how the ST-SI method compares to the two earlier methods, see $\langle 69$.

The starting point in the development of the ST-SI method was the version of the ALE-VMS method designed for computations with "sliding interfaces" $\langle 24$, 76 $>$. This ALE-VMS version has been used successfully in a number of computations with spinning solid surfaces $\langle 24$; 31, 27, 30; 76 $>$. In the ST-SI method, interface terms similar to those in the ALE-VMS version are added to the ST-VMS formulation to account for the compatibility conditions for the velocity and stress. That way, the SI between the spinning mesh and the rest of the mesh accurately connects the two sides. While having high-resolution representation of the boundary layers near a spinning solid surface, by using NURBS functions in temporal representation of the spinning motion, the ST-SI 
method can have exact representation of the circular paths and angular velocities associated with the spinning. The ST-SI method has been successfully applied to aerodynamic analysis of vertical-axis wind turbines $\langle 50\rangle$, thermo-fluid analysis of disk brakes $\langle 66>$, flow-driven string dynamics in turbomachinery $\langle 67>$, and flow analysis for the turbine part of a turbocharger $\langle 68>$. In the context of heart valves, the SI connects the sectors of meshes containing the leaflets, enabling a more effective mesh moving.

The "ST-SI-TC" method, introduced in $\langle 69>$, integrates the ST-SI and ST-TC methods in the ST-VMS framework. The initial target was to enable accurate flow analysis when we have a spinning solid surface that is in contact with a solid surface. In that context, the ST-SI-TC method was successfully applied to flow around a tire with road contact and deformation $\langle 69>$. In the context of heart valves, the ST-SI-TC method enables high-resolution representation of boundary layers even when the contact is between leaflets that are in mesh sectors connected by SIs. It also enables dealing with contact location change or contact and sliding on the SI, which of course can also be encountered in the context of spinning solid surfaces.

Compared to the finite element analysis with trilinear basis functions, Isogeometric Analysis (IGA) $<16>$ with quadratic or cubic NURBS basis functions in space was shown in $\langle 9>$ to significantly improve the solution accuracy in RBVMS computations. Computations with the ST-VMS method and ST-IGA (with NURBS basis functions in space) were first reported in $\langle 2\rangle$ in a $2 \mathrm{D}$ context. In 3D context, the ST-IGA method has been successfully applied to flow analysis of the turbine part of a turbocharger $\langle 68>$ and ram-air parachutes $\langle 70>$. In this article, we integrate the ST-SI, ST-TC and ST-IGA methods. The "ST-SI-TC-IGA," beyond enabling a more accurate representation of the surfaces and increased accuracy in the flow solution, keeps the element density in the narrow spaces near the contact areas at a reasonable level. When solid surfaces come into contact, the elements between the surface and the SI collapse. Before the elements collapse, the boundaries could be curved and rather complex, and the narrow spaces might have high-aspect-ratio elements. With 
NURBS elements, we can deal with such adverse conditions rather effectively.

Furthermore, because the flow representation in the contact area has a wider support in the ST-SI-TC-IGA, the flow computation method becomes more robust.

In Section 2 we describe the ST-VMS and ST-SI formulations. The ST-SITC-IGA method is described in Section 3 . Heart valve flow computations with an aortic-valve model and two different modes of prescribed leaflet motion are presented in Section 4. The concluding remarks are given in Section 5 .

\section{ST-VMS and ST-SI formulations}

In this section, we include from $\langle 50,69>$ the ST-VMS and ST-SI formulations.

\subsection{ST-VMS formulation}

The ST-VMS formulation is given as

$$
\begin{aligned}
& \int_{Q_{n}} \mathbf{w}^{h} \cdot \rho\left(\frac{\partial \mathbf{u}^{h}}{\partial t}+\mathbf{u}^{h} \cdot \nabla \mathbf{u}^{h}-\mathbf{f}^{h}\right) \mathrm{d} Q \\
& +\int_{Q_{n}} \varepsilon\left(\mathbf{w}^{h}\right): \boldsymbol{\sigma}\left(\mathbf{u}^{h}, p^{h}\right) \mathrm{d} Q-\int_{\left(P_{n}\right)_{\mathrm{h}}} \mathbf{w}^{h} \cdot \mathbf{h}^{h} \mathrm{~d} P \\
& +\int_{Q_{n}} q^{h} \boldsymbol{\nabla} \cdot \mathbf{u}^{h} \mathrm{~d} Q+\int_{\Omega_{n}}\left(\mathbf{w}^{h}\right)_{n}^{+} \cdot \rho\left(\left(\mathbf{u}^{h}\right)_{n}^{+}-\left(\mathbf{u}^{h}\right)_{n}^{-}\right) \mathrm{d} \Omega \\
& +\sum_{e=1}^{\left(n_{\mathrm{el}}\right)_{n}} \int_{Q_{n}^{e}} \frac{\tau_{\mathrm{SUPS}}}{\rho}\left[\rho\left(\frac{\partial \mathbf{w}^{h}}{\partial t}+\mathbf{u}^{h} \cdot \nabla_{\mathbf{w}^{h}}\right)\right. \\
& +\sum_{e=1}^{\left(n_{\mathrm{el}}\right)_{n}} \int_{Q_{n}^{e}} \nu_{\mathrm{LSIC}} \boldsymbol{\nabla} \cdot \mathbf{w}^{h} \rho r_{\mathrm{C}}\left(\mathbf{u}^{h}\right) \mathrm{d} Q \\
& \mathbf{r}_{\mathrm{M}}\left(\mathbf{u}^{h}, p^{h}\right) \mathrm{d} Q
\end{aligned}
$$


where

$$
\begin{aligned}
\mathbf{r}_{\mathrm{M}}\left(\mathbf{u}^{h}, p^{h}\right) & =\rho\left(\frac{\partial \mathbf{u}^{h}}{\partial t}+\mathbf{u}^{h} \cdot \nabla \mathbf{u}^{h}-\mathbf{f}^{h}\right)-\boldsymbol{\nabla} \cdot \boldsymbol{\sigma}\left(\mathbf{u}^{h}, p^{h}\right), \\
r_{\mathrm{C}}\left(\mathbf{u}^{h}\right) & =\nabla \cdot \mathbf{u}^{h}
\end{aligned}
$$

are the residuals of the momentum equation and incompressibility constraint. Here, $\rho, \mathbf{u}, p, \mathbf{f}, \boldsymbol{\sigma}, \boldsymbol{\varepsilon}$, and $\mathbf{h}$ are the density, velocity, pressure, external force, stress tensor, strain rate tensor, and the traction specified at the boundary. The test functions associated with the velocity and pressure are $\mathbf{w}$ and $q$. A superscript " $h$ " indicates that the function is coming from a finite-dimensional space. The symbol $Q_{n}$ represents the ST slice between time levels $n$ and $n+1$, $\left(P_{n}\right)_{\mathrm{h}}$ is the part of the lateral boundary of that slice associated with the traction boundary condition $\mathbf{h}$, and $\Omega_{n}$ is the spatial domain at time level $n$. The superscript " $e$ " is the ST element counter, and $n_{\mathrm{el}}$ is the number of ST elements. The functions are discontinuous in time at each time level, and the superscripts "-" and "+" indicate the values of the functions just below and just above the time level. See $<5,6$, 48, 65, 50> for the definitions used here for the stabilization parameters $\tau_{\text {SUPS }}$ and $\nu_{\text {LSIC }}$. For more ways of calculating the stabilization parameters in finite element computation of flow problems, see $<77$,

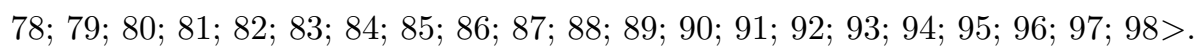

\subsection{ST-SI formulation}

\subsubsection{Two-side formulation}

Labels "Side A" and "Side B" represent the two sides of the SI. We add boundary terms to Eq. (1). The boundary terms are first added separately for the two sides, using test functions $\mathbf{w}_{\mathrm{A}}^{h}$ and $q_{\mathrm{A}}^{h}$ and $\mathbf{w}_{\mathrm{B}}^{h}$ and $q_{\mathrm{B}}^{h}$. Putting them together, the complete set of terms added becomes

$$
\begin{gathered}
-\int_{\left(P_{n}\right)_{\mathrm{SI}}}\left(q_{\mathrm{B}}^{h} \mathbf{n}_{\mathrm{B}}-q_{\mathrm{A}}^{h} \mathbf{n}_{\mathrm{A}}\right) \cdot \frac{1}{2}\left(\mathbf{u}_{\mathrm{B}}^{h}-\mathbf{u}_{\mathrm{A}}^{h}\right) \mathrm{d} P \\
-\int_{\left(P_{n}\right)_{\mathrm{SI}}} \rho \mathbf{w}_{\mathrm{B}}^{h} \cdot \frac{1}{2}\left(\left(\mathcal{F}_{\mathrm{B}}^{h}-\left|\mathcal{F}_{\mathrm{B}}^{h}\right|\right) \mathbf{u}_{\mathrm{B}}^{h}\right. \\
\left.-\left(\mathcal{F}_{\mathrm{B}}^{h}-\left|\mathcal{F}_{\mathrm{B}}^{h}\right|\right) \mathbf{u}_{\mathrm{A}}^{h}\right) \mathrm{d} P
\end{gathered}
$$




$$
\begin{aligned}
& -\int_{\left(P_{n}\right)_{\mathrm{SI}}} \rho \mathbf{w}_{\mathrm{A}}^{h} \cdot \frac{1}{2}\left(\left(\mathcal{F}_{\mathrm{A}}^{h}-\left|\mathcal{F}_{\mathrm{A}}^{h}\right|\right) \mathbf{u}_{\mathrm{A}}^{h}\right. \\
& \left.-\left(\mathcal{F}_{\mathrm{A}}^{h}-\left|\mathcal{F}_{\mathrm{A}}^{h}\right|\right) \mathbf{u}_{\mathrm{B}}^{h}\right) \mathrm{d} P \\
& +\int_{\left(P_{n}\right)_{\mathrm{SI}}}\left(\mathbf{n}_{\mathrm{B}} \cdot \mathbf{w}_{\mathrm{B}}^{h}+\mathbf{n}_{\mathrm{A}} \cdot \mathbf{w}_{\mathrm{A}}^{h}\right) \frac{1}{2}\left(p_{\mathrm{B}}^{h}+p_{\mathrm{A}}^{h}\right) \mathrm{d} P \\
& -\int_{\left(P_{n}\right)_{\mathrm{SI}}}\left(\mathbf{w}_{\mathrm{B}}^{h}-\mathbf{w}_{\mathrm{A}}^{h}\right) \cdot\left(\hat{\mathbf{n}}_{\mathrm{B}} \cdot \mu\left(\varepsilon\left(\mathbf{u}_{\mathrm{B}}^{h}\right)+\boldsymbol{\varepsilon}\left(\mathbf{u}_{\mathrm{A}}^{h}\right)\right) \mathrm{d} P\right. \\
& -\gamma \int_{\left(P_{n}\right)_{\mathrm{SI}}} \hat{\mathbf{n}}_{\mathrm{B}} \cdot \mu\left(\varepsilon\left(\mathbf{w}_{\mathrm{B}}^{h}\right)+\varepsilon\left(\mathbf{w}_{\mathrm{A}}^{h}\right)\right) \cdot\left(\mathbf{u}_{\mathrm{B}}^{h}-\mathbf{u}_{\mathrm{A}}^{h}\right) \mathrm{d} P \\
& +\int_{\left(P_{n}\right)_{\mathrm{SI}}} \frac{\mu C}{h}\left(\mathbf{w}_{\mathrm{B}}^{h}-\mathbf{w}_{\mathrm{A}}^{h}\right) \cdot\left(\mathbf{u}_{\mathrm{B}}^{h}-\mathbf{u}_{\mathrm{A}}^{h}\right) \mathrm{d} P,
\end{aligned}
$$

where

$$
\begin{aligned}
\mathcal{F}_{\mathrm{B}}^{h} & =\mathbf{n}_{\mathrm{B}} \cdot\left(\mathbf{u}_{\mathrm{B}}^{h}-\mathbf{v}_{\mathrm{B}}^{h}\right), \\
\mathcal{F}_{\mathrm{A}}^{h} & =\mathbf{n}_{\mathrm{A}} \cdot\left(\mathbf{u}_{\mathrm{A}}^{h}-\mathbf{v}_{\mathrm{A}}^{h}\right), \\
h & =\frac{h_{\mathrm{B}}+h_{\mathrm{A}}}{2}, \\
h_{\mathrm{B}} & =2\left(\sum_{\alpha=1}^{n_{\text {ent }}} \sum_{a=1}^{n_{\text {ens }}}\left|\mathbf{n}_{\mathrm{B}} \cdot \nabla N_{a}^{\alpha}\right|\right)^{-1} \text { (for Side B), } \\
h_{\mathrm{A}} & =2\left(\sum_{\alpha=1}^{n_{\text {ent }}} \sum_{a=1}^{n_{\text {ens }}}\left|\mathbf{n}_{\mathrm{A}} \cdot \nabla N_{a}^{\alpha}\right|\right)^{-1} \text { (for Side A), } \\
\hat{\mathbf{n}}_{\mathrm{B}} & =\frac{\mathbf{n}_{\mathrm{B}}-\mathbf{n}_{\mathrm{A}}}{\left\|\mathbf{n}_{\mathrm{B}}-\mathbf{n}_{\mathrm{A}}\right\|} .
\end{aligned}
$$

Here, $\left(P_{n}\right)_{\mathrm{SI}}$ is the SI in the ST domain, $\mathbf{n}$ is the unit normal vector, $\mathbf{v}$ is the mesh velocity, $n_{\mathrm{ens}}$ and $n_{\mathrm{ent}}$ are the number of spatial and temporal element nodes, $N_{a}^{\alpha}$ is the basis function associated with spatial and temporal nodes $a$ and $\alpha, \gamma=1$, and $C$ is a nondimensional constant. For our element length definition, we set $C=1$.

\subsubsection{One-side formulation}

Sometimes we prefer to specify on solid surfaces weakly-imposed Dirichlet conditions for the fluid $\langle 99$; 23$\rangle$. In such cases we use the ST-SI version where the SI is between the fluid and solid domains. This version is obtained (see $<\mid 50>$ ) by starting with the terms added for Side B and replacing the Side A 
velocity with the velocity $\mathrm{g}^{h}$ coming from the solid domain. Then the terms added to Eq. (1) to represent the weakly-imposed Dirichlet conditions become

$$
\begin{aligned}
& -\int_{\left(P_{n}\right)_{\mathrm{SI}}} q_{\mathrm{B}}^{h} \mathbf{n}_{\mathrm{B}} \cdot \mathbf{u}_{\mathrm{B}}^{h} \mathrm{~d} P-\int_{\left(P_{n}\right)_{\mathrm{SI}}} \rho \mathbf{w}_{\mathrm{B}}^{h} \cdot \mathcal{F}_{\mathrm{B}}^{h} \mathbf{u}_{\mathrm{B}}^{h} \mathrm{~d} P \\
& +\int_{\left(P_{n}\right)_{\mathrm{SI}}} q_{\mathrm{B}}^{h} \mathbf{n}_{\mathrm{B}} \cdot \mathbf{g}^{h} \mathrm{~d} P \\
& +\int_{\left(P_{n}\right)_{\mathrm{SI}}} \rho \mathbf{w}_{\mathrm{B}}^{h} \cdot \frac{1}{2}\left(\left(\mathcal{F}_{\mathrm{B}}^{h}+\left|\mathcal{F}_{\mathrm{B}}^{h}\right|\right) \mathbf{u}_{\mathrm{B}}^{h}\right. \\
& \left.\quad+\left(\mathcal{F}_{\mathrm{B}}^{h}-\left|\mathcal{F}_{\mathrm{B}}^{h}\right|\right) \mathbf{g}^{h}\right) \mathrm{d} P \\
& -\int_{\left(P_{n}\right)_{\mathrm{SI}}} \mathbf{w}_{\mathrm{B}}^{h} \cdot\left(\mathbf{n}_{\mathrm{B}} \cdot \boldsymbol{\sigma}_{\mathrm{B}}^{h}\right) \mathrm{d} P \\
& -\gamma \int_{\left(P_{n}\right)_{\mathrm{SI}}} \mathbf{n}_{\mathrm{B}} \cdot 2 \mu \varepsilon\left(\mathbf{w}_{\mathrm{B}}^{h}\right) \cdot\left(\mathbf{u}_{\mathrm{B}}^{h}-\mathbf{g}^{h}\right) \mathrm{d} P \\
& +\int_{\left(P_{n}\right)_{\mathrm{SI}}} \frac{\mu C}{h_{\mathrm{B}}} \mathbf{w}_{\mathrm{B}}^{h} \cdot\left(\mathbf{u}_{\mathrm{B}}^{h}-\mathbf{g}^{h}\right) \mathrm{d} P .
\end{aligned}
$$

\section{ST-SI-TC-IGA method}

We first overview the aspects of the ST-SI $<50>$ and ST-TC $<55>>$ methods relevant to their integration as the ST-SI-TC method $\langle 69>$, and then describe the advantages of the IGA in this context and integration of all three components as the ST-SI-TC-IGA method.

\subsection{ST-SI method}

We note that the ST-SI method allows mesh slipping also in the one-side formulation, that is, when the SI is between the fluid and solid domains where we have weakly-imposed Dirichlet conditions. The boundary terms added to Eq. (1) to connect the two sides and to connect the fluid to the solid in the one-side formulation were given in Sections 2.2.1 and 2.2.2. The added terms (see Eqs. (4) and (11) include derivatives in the direction normal to the SI. Therefore the elements bordering the SI need to have finite thickness in the normal direction. This places a limitation on the meshes that can be used with the ST-SI method because if an element bordering the interface degenerates it might lead to a zero element thickness in the normal direction. 


\section{2. $S T$-TC method}

The ST-TC method can deal with TC in ST moving-mesh computations. The discretization is unstructured in time, but based on a "parent" mesh that is structured in time. The ST parent mesh is extruded from a single spatial mesh. The key technology is massive element degeneration by using a special masterslave system. This special system allows changing, within an ST slab, master nodes to slave nodes and slave nodes to master nodes. With that, elements can collapse or be reborn. This way, within an ST slab, we can represent closing and opening motions. Since an ST method naturally allows discretizations that are unstructured in time, the rest of the method needs no modification. The method is very flexible, and computationally as effective as a typical movingmesh method. However, the master-slave relationship has to be node to node; a point on a solid surface that is not a node cannot be a master or slave node.

\subsection{ST-IGA method}

With NURBS meshes, we can represent curved boundaries with less elements compared to finite element meshes. With this desirable feature, a volume can also be meshed with high aspect ratio elements. This is particularly helpful when we need to generate meshes in very narrow spaces.

\subsection{Integration of the ST-SI, ST-TC and ST-IGA methods}

Integration of these three methods brings a number of advantages. (i) It enables high-resolution boundary layer representation near the solid surfaces in contact even when the surfaces are covered by meshes with SI. (ii) It enables dealing with contact location change or contact and sliding on the SI. This overcomes the ST-TC restriction that a point on a solid surface that is not a node cannot be a master or slave node. (iii) When surfaces contact each other, the elements between the surface and the SI collapse. Before the elements collapse, the boundaries could be curved and complex, and the narrow space might have high-aspect-ratio elements. With NURBS elements, we can deal with such adverse conditions rather effectively. We note that NURBS basis 
functions of higher order and smoothness, in contrast to standard, low-order finite element basis functions, can withstand higher levels of mesh distortion and degeneration and maintain good approximation properties. (iv) Because the flow representation in the contact area has a wider support in IGA, the flow computation method becomes more robust.

To illustrate how the ST-SI-TC-IGA method works, we show with a 2D example how we deal with two valve leaflets flapping and coming into contact in an asymmetric fashion. Figure 1 shows the mesh motion, and Figure 2 shows the corresponding control mesh.

Remark 1. As can be seen in Figure 1, we have high-aspect-ratio elements near the contact region, and the NURBS elements can deal with that without mesh entanglement.

Remark 2. We allow situations where a surface from only one of the two sides coincides with the SI. This gives us the freedom of the two sides of the SI having their own master-slave relationships. For the surface that coincides with the SI, the SI serves the purpose of enforcing the weak Dirichlet condition.

Remark 3. In the case of quadratic NURBS elements, three control points along each of the two parametric directions aligned with the SI must coalesce with the corresponding control points in the third direction to result in a zerovolume element (see Figure 2 for the $2 D$ version of that). Unless that happens, the element is treated as an element with nonzero volume, and the element integrations are performed using all the integration points of the element. We also note that even if a spatial element belonging to an $S T$ element has zero volume, as long as the ST element has nonzero volume, we integrate over that ST element (see $<55>$ for details). 



Figure 1: An example of contact and sliding (element representation). Element colors indicate the NURBS patches the elements belong to. The thick red curves are the leaflets. The two green vertical lines, with the narrow space between them added only for illustration purposes, represent the two sides of an SI. 

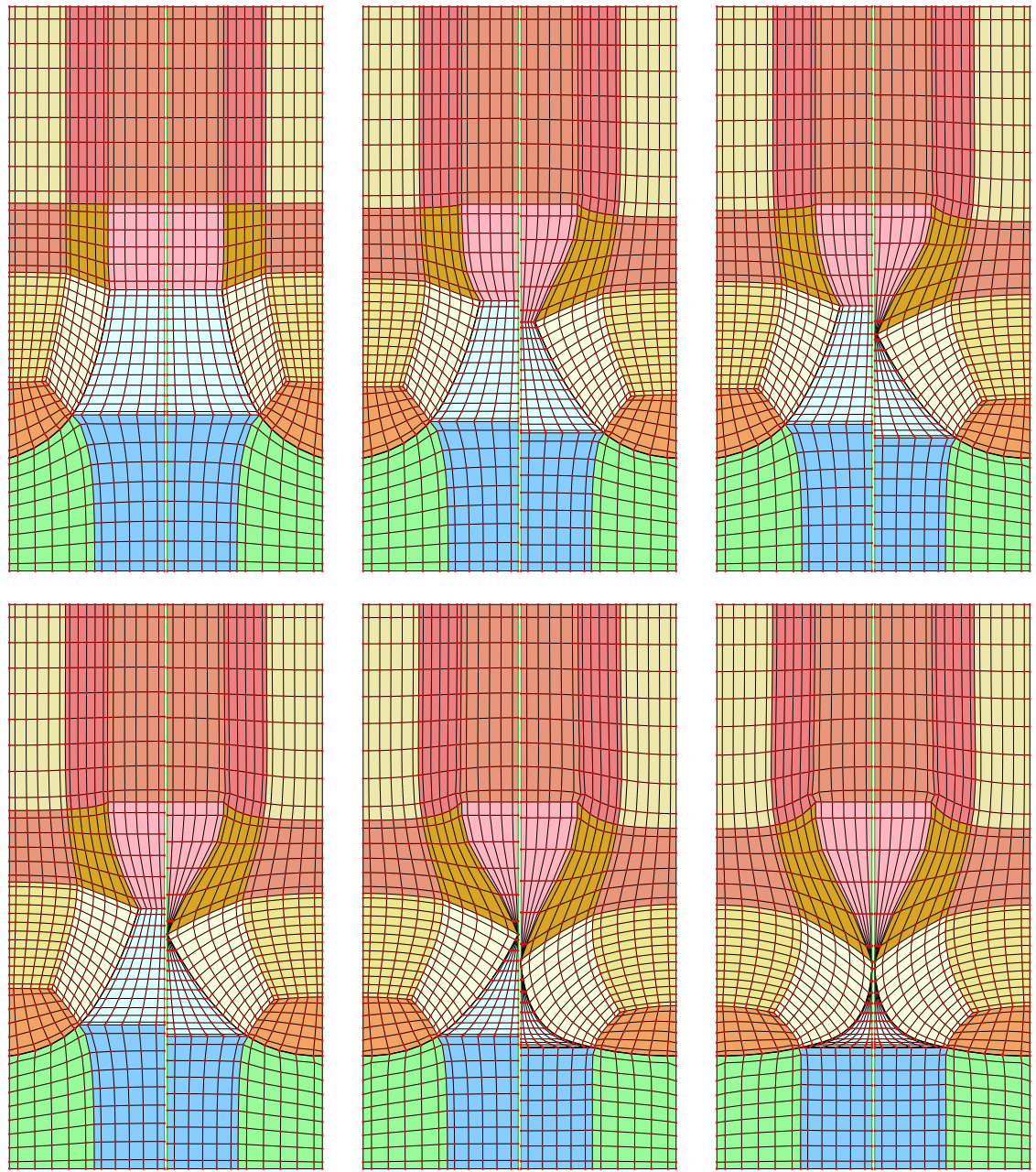

Figure 2: An example of contact and sliding (control mesh). Shading colors indicate the NURBS patches. The black curves, which are actually collections of line segments, represent the control-mesh surfaces corresponding to the leaflets. The control points on the two sides of the narrow vertical gap correspond to the two sides of the SI. When control points coincide, they are in a master-slave relationship. 


\section{Aortic-valve model}

\subsection{Geometry}

We have a typical aortic-valve model, such as the one in $\angle 57>$. The model, shown in Figure 3 , has three leaflets and one main outlet, corresponding to the beginning of the aorta. The leaflet motion is prescribed.

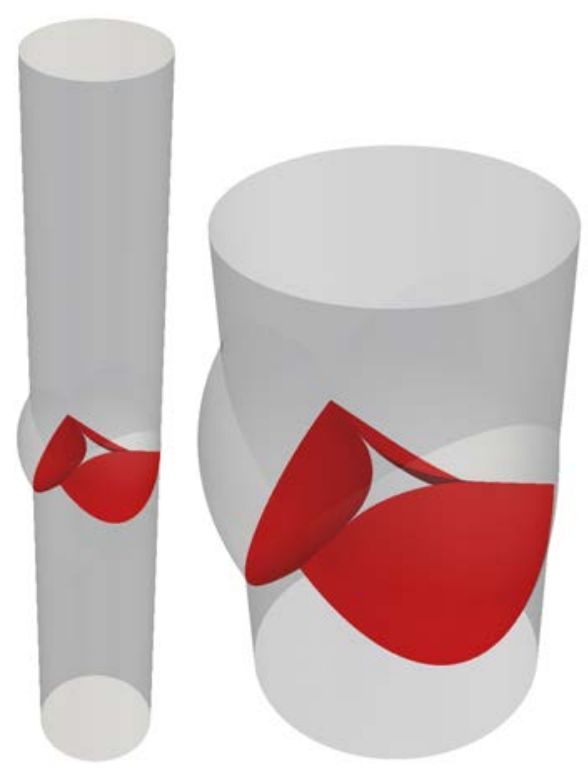

Figure 3: Aortic-valve model geometry. Aorta, leaflets, and sinuses. The left picture shows the entire computational domain, and the right picture is the zoomed view of the valve.

\subsection{Leaflet motion}

We consider two cases of leaflet motion: symmetric and asymmetric. In the symmetric case, the leaflet motions have 3-fold rotational periodicity (i.e., 3 -fold discrete rotational symmetry), and in the asymmetric case, they do not. We identify the individual leaflets as shown in Figure 4. The leaflet positions are defined by means of a pseudo-time parameter $\theta$, with the values 0 and 1 corresponding to the fully open and fully closed positions. The prescribed motion is given through $\theta$ as shown in Figure 5 for the symmetric case, and in Figure 6 for the asymmetric case. 


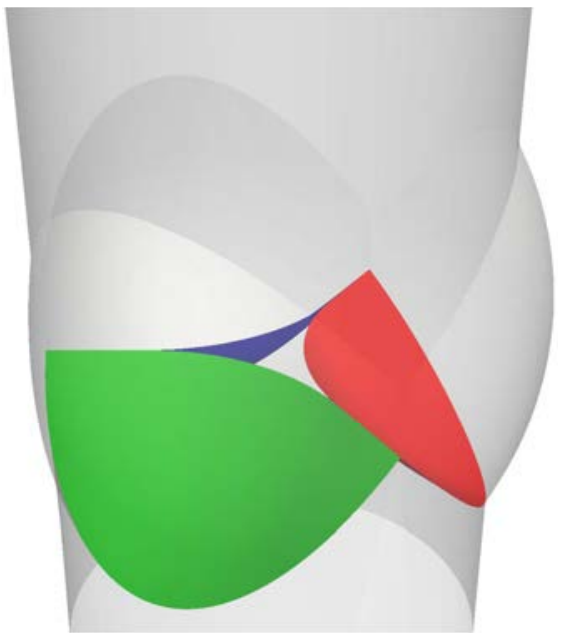

Figure 4: Leaflet identification. Leaflet 1 (red), 2 (green) and 3 (blue).

\subsection{Mesh and flow conditions}

We create the mesh with five SIs, with three of them connecting the mesh sectors containing the leaflets in the valve region of the aorta (see Figure 7). The other two SIs, which are the top and bottom circular planes in Figure 7 connect the meshes in the inlet and outlet regions to the valve region. They are for independent meshing in the inlet and outlet regions.

The volume mesh is made of quadratic NURBS elements. The number of control points is 84,534 , and the number of elements is 54,000 . We prescribe the motion of the interior control points, and specify in each domain the masterslave mapping for all leaflet positions. Figure 8 shows a set of selected NURBS elements to illustrate how elements collapse.

The boundary conditions are no-slip on the arterial walls and the leaflets, traction-free at the outflow boundary, and uniform velocity at the inflow boundary, with a temporal profile as shown in Figure 9. The cycle period is $0.712 \mathrm{~s}$. The no-slip condition on the arterial walls is based on the weakly-imposed Dirichlet condition given by Eq. 11. 


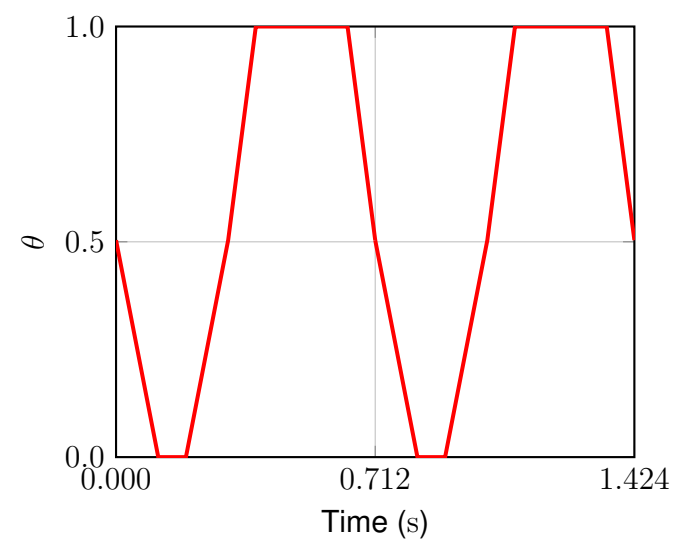

Figure 5: Symmetric leaflet motion. Pseudo-time parameter $\theta$ as a function of time for each of the three leaflets.

\subsection{Computational conditions}

We use the ST-SUPS method, which can obtained by dropping the last two series of integrations in Eq. (1). The time-step size is $4.00 \times 10^{-3} \mathrm{~s}$. There are three nonlinear iterations at each time step. The number of GMRES iterations per nonlinear iteration is 300 .

\subsection{Results}

\subsubsection{Symmetric leaflet motion}

Figures 10 and 11 show the isosurfaces corresponding to a positive value of the second invariant of the velocity gradient tensor, colored by the velocity magnitude. The viewing angle is as we see the leaflets in Figure 4 The result shows how effectively the flow separation is captured near the leaflet edges and the solution is obtained as the surfaces come into contact.

Remark 4. We note that there is a thin vortex sheet near the aorta wall in the second and third row of pictures in Figure 11, which correspond to the fully closed positions of the valve. That is because full blocking of the flow requires a collapsed (i.e. zero-volume) element, which in turn requires that the control points of the element coalesce as described in Remark 3, and that is not happening for the 


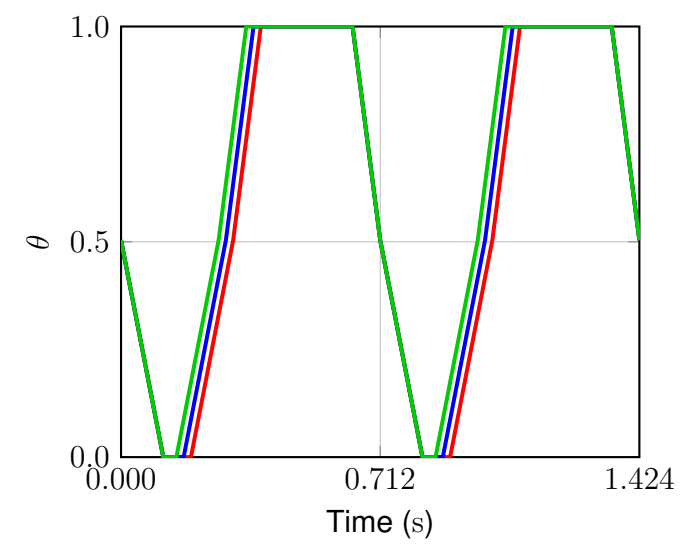

Figure 6: Asymmetric leaflet motion. Pseudo-time parameter $\theta$ as a function of time for each of the three leaflets.

elements with two edges on the leaflet edges and two edges on the aorta wall. In the current setting, the control points associated with the two edges on the aorta wall cannot coalesce.

We also report the the wall shear stress (WSS) on the leaflet surfaces. The viewing angle is as we see the leaflets in Figure 12 . Figures 13 and 14 show the magnitude of the WSS on the upper and lower surfaces of the leaflets. As can be seen in the figures, the ST-SI-TC-IGA method gives a smooth WSS distribution, and the upper and lower WSS distributions are unrelated. The WSS is high on the lower surface near the edge, which is due to the flow jet.

\subsubsection{Asymmetric leaflet motion}

Figures 15 and 16 show the isosurfaces corresponding to a positive value of the second invariant of the velocity gradient tensor, colored by the velocity magnitude. The viewing angle is as we see the leaflets in Figure 4. In this case we have a biased flow jet due to the asymmetric leaflet closing. This can be seen from the fifth row of pictures in Figure 15 and the first and second row of pictures in Figure 16 .

Figures 17 and 18 show the magnitude of the WSS on the upper and lower surfaces of the leaflets. The viewing angle is as we see the leaflets in Figure 12 


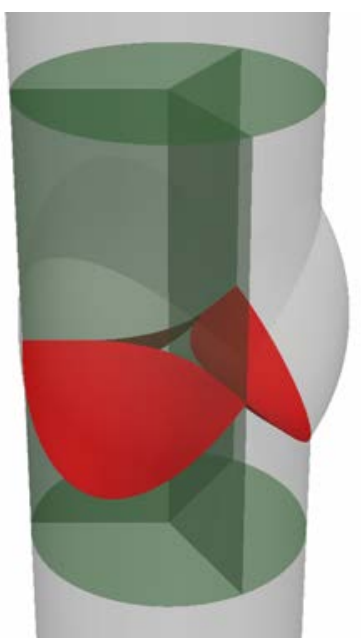

Figure 7: Aortic valve and the five SIs.

\section{Concluding remarks}

Heart valve flow computation requires dealing successfully with a high level of geometric complexity and representing accurately the boundary layers near moving solid surfaces, including the valve leaflet surfaces, even when the leaflets come into contact. We have addressed these computational challenges with an ST method that is composed of three special ST methods, integrated in the framework of the ST-VMS method. The special methods are the STSI and ST-TC methods and ST-IGA. The computations were for a realistic aortic-valve model with prescribed valve leaflet motion and actual contact between the leaflets. The ST-VMS method functions as a moving-mesh method, which maintains high-resolution boundary layer representation near the solid surfaces, including the leaflet surfaces. The ST-TC method was introduced for moving-mesh computation of flow problems with TC, such as contact between the leaflets of a heart valve. It deals with the contact while maintaining high-resolution representation near the leaflet surfaces. The ST-SI method was originally introduced to have high-resolution representation of the boundary 
layers near spinning solid surfaces. The mesh covering a spinning solid surface spins with it, and the SI between the spinning mesh and the rest of the mesh accurately connects the two sides. This maintains the high-resolution representation near solid surfaces. In the context of heart valves, the SI connects the sectors of meshes containing the leaflets, giving us more freedom in mesh generation and mesh moving, and that helps us deal with the high level of geometric complexity. In that context, integration of the ST-SI and ST-TC methods enables high-resolution representation of boundary layers near leaflet surfaces even when the contact is between leaflets that are covered by meshes with SI. It also enables dealing with contact location change or contact and sliding on the SI. By integrating the ST-IGA with the ST-SI and ST-TC methods, in addition to having a more accurate representation of the surfaces and increased accuracy in the flow solution, the element density in the narrow spaces near the contact areas is kept at a reasonable level. When solid surfaces come into contact, before the elements between the surface and the SI collapse, the boundaries could be curved and rather complex, and the narrow spaces might have high-aspect-ratio elements. The ST-SI-TC-IGA method can deal with such adverse conditions rather effectively. Furthermore, because the flow representation in the contact area has a wider support in the ST-SI-TC-IGA method, we have a flow computation method with increased robustness. With the computations we have presented, which were for two different modes of leaflet motion, we have shown the effectiveness of the ST-SI-TC-IGA method.

\section{Acknowledgement}

This work was supported (first, third, and fourth authors) in part by JSTCREST; Grant-in-Aid for Young Scientists (B) 24760144 from Japan Society for the Promotion of Science (JSPS); Grant-in-Aid for Scientific Research (S) 26220002 from the Ministry of Education, Culture, Sports, Science and Technology of Japan (MEXT); and Rice-Waseda research agreement.

[1] K. Takizawa, T.E. Tezduyar, T. Terahara, and T. Sasaki, "Heart valve flow 
computation with the Space-Time Slip Interface Topology Change (STSI-TC) method and Isogeometric Analysis (IGA)", to appear in a special volume to be published by Springer, 2016 .

[2] K. Takizawa and T.E. Tezduyar, "Multiscale space-time fluid-structure interaction techniques", Computational Mechanics, 48 (2011) 247-267, doi: 10.1007/s00466-011-0571-z.

[3] K. Takizawa and T.E. Tezduyar, "Space-time fluid-structure interaction methods", Mathematical Models and Methods in Applied Sciences, 22 (supp02) (2012) 1230001, doi: 10.1142/S0218202512300013.

[4] T.E. Tezduyar, "Stabilized finite element formulations for incompressible flow computations", Advances in Applied Mechanics, 28 (1992) 1-44, doi: 10.1016/S0065-2156(08)70153-4.

[5] T.E. Tezduyar, "Computation of moving boundaries and interfaces and stabilization parameters", International Journal for Numerical Methods in Fluids, 43 (2003) 555-575, doi: 10.1002/fld.505.

[6] T.E. Tezduyar and S. Sathe, "Modeling of fluid-structure interactions with the space-time finite elements: Solution techniques", International Journal for Numerical Methods in Fluids, 54 (2007) 855-900, doi: 10.1002/fld.1430.

[7] T.J.R. Hughes, "Multiscale phenomena: Green's functions, the Dirichletto-Neumann formulation, subgrid scale models, bubbles, and the origins of stabilized methods", Computer Methods in Applied Mechanics and Engineering, 127 (1995) 387-401.

[8] T.J.R. Hughes, A.A. Oberai, and L. Mazzei, "Large eddy simulation of turbulent channel flows by the variational multiscale method", Physics of Fluids, 13 (2001) 1784-1799.

[9] Y. Bazilevs, V.M. Calo, J.A. Cottrell, T.J.R. Hughes, A. Reali, and G. Scovazzi, "Variational multiscale residual-based turbulence modeling for large 
eddy simulation of incompressible flows", Computer Methods in Applied Mechanics and Engineering, 197 (2007) 173-201.

[10] Y. Bazilevs and I. Akkerman, "Large eddy simulation of turbulent TaylorCouette flow using isogeometric analysis and the residual-based variational multiscale method", Journal of Computational Physics, 229 (2010) 34023414.

[11] A.N. Brooks and T.J.R. Hughes, "Streamline upwind/Petrov-Galerkin formulations for convection dominated flows with particular emphasis on the incompressible Navier-Stokes equations", Computer Methods in Applied Mechanics and Engineering, 32 (1982) 199-259.

[12] Y. Bazilevs, V.M. Calo, T.J.R. Hughes, and Y. Zhang, "Isogeometric fluidstructure interaction: theory, algorithms, and computations", Computational Mechanics, 43 (2008) 3-37.

[13] K. Takizawa, Y. Bazilevs, and T.E. Tezduyar, "Space-time and ALEVMS techniques for patient-specific cardiovascular fluid-structure interaction modeling", Archives of Computational Methods in Engineering, 19 (2012) 171-225, doi: 10.1007/s11831-012-9071-3.

[14] Y. Bazilevs, M.-C. Hsu, K. Takizawa, and T.E. Tezduyar, "ALEVMS and ST-VMS methods for computer modeling of wind-turbine rotor aerodynamics and fluid-structure interaction", Mathematical Models and Methods in Applied Sciences, 22 (supp02) (2012) 1230002, doi: 10.1142/S0218202512300025.

[15] T.J.R. Hughes, W.K. Liu, and T.K. Zimmermann, "Lagrangian-Eulerian finite element formulation for incompressible viscous flows", Computer Methods in Applied Mechanics and Engineering, 29 (1981) 329-349.

[16] T.J.R. Hughes, J.A. Cottrell, and Y. Bazilevs, "Isogeometric analysis: CAD, finite elements, NURBS, exact geometry, and mesh re- 
finement", Computer Methods in Applied Mechanics and Engineering, 194 (2005) 4135-4195.

[17] Y. Bazilevs, V.M. Calo, Y. Zhang, and T.J.R. Hughes, "Isogeometric fluidstructure interaction analysis with applications to arterial blood flow", Computational Mechanics, 38 (2006) 310-322.

[18] Y. Bazilevs and T.J.R. Hughes, "NURBS-based isogeometric analysis for the computation of flows about rotating components", Computational Mechanics, 43 (2008) 143-150.

[19] Y. Bazilevs, J.R. Gohean, T.J.R. Hughes, R.D. Moser, and Y. Zhang, "Patient-specific isogeometric fluid-structure interaction analysis of thoracic aortic blood flow due to implantation of the Jarvik 2000 left ventricular assist device", Computer Methods in Applied Mechanics and Engineering, 198 (2009) 3534-3550.

[20] Y. Bazilevs, M.-C. Hsu, D. Benson, S. Sankaran, and A. Marsden, "Computational fluid-structure interaction: Methods and application to a total cavopulmonary connection", Computational Mechanics, 45 (2009) 77-89.

[21] Y. Bazilevs, M.-C. Hsu, I. Akkerman, S. Wright, K. Takizawa, B. Henicke, T. Spielman, and T.E. Tezduyar, "3D simulation of wind turbine rotors at full scale. Part I: Geometry modeling and aerodynamics", International Journal for Numerical Methods in Fluids, 65 (2011) 207-235, doi: 10.1002/fld.2400.

[22] Y. Bazilevs, M.-C. Hsu, J. Kiendl, R. Wüchner, and K.-U. Bletzinger, "3D simulation of wind turbine rotors at full scale. Part II: Fluid-structure interaction modeling with composite blades", International Journal for $\mathrm{Nu}$ merical Methods in Fluids, 65 (2011) 236-253.

[23] M.-C. Hsu, I. Akkerman, and Y. Bazilevs, "Wind turbine aerodynamics using ALE-VMS: Validation and role of weakly enforced boundary conditions", Computational Mechanics, 50 (2012) 499-511. 
[24] M.-C. Hsu and Y. Bazilevs, "Fluid-structure interaction modeling of wind turbines: simulating the full machine", Computational Mechanics, 50 (2012) 821-833.

[25] Y. Bazilevs, K. Takizawa, and T.E. Tezduyar, Computational FluidStructure Interaction: Methods and Applications. Wiley, February 2013, ISBN 978-0470978771.

[26] Y. Bazilevs, K. Takizawa, and T.E. Tezduyar, "Challenges and directions in computational fluid-structure interaction", Mathematical Models and Methods in Applied Sciences, 23 (2013) 215-221, doi: 10.1142/S0218202513400010.

[27] A. Korobenko, M.-C. Hsu, I. Akkerman, J. Tippmann, and Y. Bazilevs, "Structural mechanics modeling and FSI simulation of wind turbines", Mathematical Models and Methods in Applied Sciences, 23 (2013) 249-272.

[28] A. Korobenko, M.-C. Hsu, I. Akkerman, and Y. Bazilevs, "Aerodynamic simulation of vertical-axis wind turbines", Journal of Applied Mechanics, 81 (2013) 021011, doi: 10.1115/1.4024415.

[29] Y. Bazilevs, K. Takizawa, T.E. Tezduyar, M.-C. Hsu, N. Kostov, and S. McIntyre, "Aerodynamic and FSI analysis of wind turbines with the ALE-VMS and ST-VMS methods", Archives of Computational Methods in Engineering, 21 (2014) 359-398, doi: 10.1007/s11831-014-9119-7.

[30] Y. Bazilevs, A. Korobenko, X. Deng, J. Yan, M. Kinzel, and J.O. Dabiri, "FSI modeling of vertical-axis wind turbines", Journal of Applied Mechanics, 81 (2014) 081006, doi: 10.1115/1.4027466.

[31] M.-C. Hsu, I. Akkerman, and Y. Bazilevs, "Finite element simulation of wind turbine aerodynamics: Validation study using NREL Phase VI experiment", Wind Energy, 17 (2014) 461-481.

[32] C.C. Long, M. Esmaily-Moghadam, A.L. Marsden, and Y. Bazilevs, "Computation of residence time in the simulation of pulsatile ventric- 
ular assist devices", Computational Mechanics, 54 (2014) 911-919, doi: 10.1007/s00466-013-0931-y.

[33] C.C. Long, A.L. Marsden, and Y. Bazilevs, "Shape optimization of pulsatile ventricular assist devices using FSI to minimize thrombotic risk", Computational Mechanics, 54 (2014) 921-932, doi: 10.1007/s00466-013-0967-z.

[34] M.-C. Hsu, D. Kamensky, Y. Bazilevs, M.S. Sacks, and T.J.R. Hughes, "Fluid-structure interaction analysis of bioprosthetic heart valves: significance of arterial wall deformation", Computational Mechanics, 54 (2014) 1055-1071, doi: 10.1007/s00466-014-1059-4.

[35] B. Augier, J. Yan, A. Korobenko, J. Czarnowski, G. Ketterman, and Y. Bazilevs, "Experimental and numerical FSI study of compliant hydrofoils", Computational Mechanics, 55 (2015) 1079-1090, doi: 10.1007/s00466-014-1090-5.

[36] Y. Bazilevs, A. Korobenko, J. Yan, A. Pal, S.M.I. Gohari, and S. Sarkar, "ALE-VMS formulation for stratified turbulent incompressible flows with applications", Mathematical Models and Methods in Applied Sciences, 25 (2015) 2349-2375, doi: 10.1142/S0218202515400114.

[37] Y. Bazilevs, K. Takizawa, and T.E. Tezduyar, "New directions and challenging computations in fluid dynamics modeling with stabilized and multiscale methods", Mathematical Models and Methods in Applied Sciences, 25 (2015) 2217-2226, doi: 10.1142/S0218202515020029.

[38] Y. Bazilevs, A. Korobenko, X. Deng, and J. Yan, "FSI modeling for fatiguedamage prediction in full-scale wind-turbine blades", Journal of Applied Mechanics, 83 (6) (2016) 061010.

[39] J. Yan, B. Augier, A. Korobenko, J. Czarnowski, G. Ketterman, and Y. Bazilevs, "FSI modeling of a propulsion system based on compliant hydrofoils in a tandem configuration", Computers and Fluids, (2015), Published online, doi:10.1016/j.compfluid.2015.07.013. 
[40] J. Yan, A. Korobenko, X. Deng, and Y. Bazilevs, "Computational free-surface fluid-structure interaction with application to floating offshore wind turbines", Computers $\&$ Fluids, (2016), Published online, doi:10.1016/j.compfluid.2016.03.008.

[41] K. Takizawa, Y. Bazilevs, T.E. Tezduyar, M.-C. Hsu, O. Øiseth, K.M. Mathisen, N. Kostov, and S. McIntyre, "Engineering analysis and design with ALE-VMS and space-time methods", Archives of Computational Methods in Engineering, 21 (2014) 481-508, doi: 10.1007/s11831-014-91130.

[42] K. Takizawa, T.E. Tezduyar, R. Kolesar, C. Boswell, T. Kanai, and K. Montel, "Multiscale methods for gore curvature calculations from FSI modeling of spacecraft parachutes", Computational Mechanics, 54 (2014) 1461-1476, doi: 10.1007/s00466-014-1069-2.

[43] K. Takizawa, T.E. Tezduyar, C. Boswell, R. Kolesar, and K. Montel, "FSI modeling of the reefed stages and disreefing of the Orion spacecraft parachutes", Computational Mechanics, 54 (2014) 1203-1220, doi: 10.1007/s00466-014-1052-y.

[44] K. Takizawa, T.E. Tezduyar, C. Boswell, Y. Tsutsui, and K. Montel, "Special methods for aerodynamic-moment calculations from parachute FSI modeling", Computational Mechanics, 55 (2015) 1059-1069, doi: 10.1007/s00466-014-1074-5.

[45] K. Takizawa, T.E. Tezduyar, and R. Kolesar, "FSI modeling of the Orion spacecraft drogue parachutes", Computational Mechanics, 55 (2015) 11671179, doi: 10.1007/s00466-014-1108-z.

[46] K. Takizawa, B. Henicke, T.E. Tezduyar, M.-C. Hsu, and Y. Bazilevs, "Stabilized space-time computation of wind-turbine rotor aerodynamics", Computational Mechanics, 48 (2011) 333-344, doi: 10.1007/s00466-0110589-2, 
[47] K. Takizawa, B. Henicke, D. Montes, T.E. Tezduyar, M.-C. Hsu, and Y. Bazilevs, "Numerical-performance studies for the stabilized space-time computation of wind-turbine rotor aerodynamics", Computational Mechanics, 48 (2011) 647-657, doi: 10.1007/s00466-011-0614-5.

[48] K. Takizawa, T.E. Tezduyar, S. McIntyre, N. Kostov, R. Kolesar, and C. Habluetzel, "Space-time VMS computation of wind-turbine rotor and tower aerodynamics", Computational Mechanics, 53 (2014) 1-15, doi: 10.1007/s00466-013-0888-x.

[49] K. Takizawa, "Computational engineering analysis with the new-generation space-time methods", Computational Mechanics, 54 (2014) 193-211, doi: 10.1007/s00466-014-0999-z.

[50] K. Takizawa, T.E. Tezduyar, H. Mochizuki, H. Hattori, S. Mei, L. Pan, and K. Montel, "Space-time VMS method for flow computations with slip interfaces (ST-SI)", Mathematical Models and Methods in Applied Sciences, 25 (2015) 2377-2406, doi: 10.1142/S0218202515400126.

[51] K. Takizawa, B. Henicke, A. Puntel, T. Spielman, and T.E. Tezduyar, "Space-time computational techniques for the aerodynamics of flapping wings", Journal of Applied Mechanics, 79 (2012) 010903, doi: $10.1115 / 1.4005073$.

[52] K. Takizawa, B. Henicke, A. Puntel, N. Kostov, and T.E. Tezduyar, "Space-time techniques for computational aerodynamics modeling of flapping wings of an actual locust", Computational Mechanics, 50 (2012) 743760, doi: $10.1007 / \mathrm{s} 00466-012-0759-\mathrm{x}$

[53] K. Takizawa, N. Kostov, A. Puntel, B. Henicke, and T.E. Tezduyar, "Space-time computational analysis of bio-inspired flapping-wing aerodynamics of a micro aerial vehicle", Computational Mechanics, 50 (2012) 761778, doi: $10.1007 / \mathrm{s} 00466-012-0758-y$. 
[54] K. Takizawa, B. Henicke, A. Puntel, N. Kostov, and T.E. Tezduyar, "Computer modeling techniques for flapping-wing aerodynamics of a locust", Computers 85 Fluids, 85 (2013) 125-134, doi: 10.1016/j.compfluid.2012.11.008.

[55] K. Takizawa, T.E. Tezduyar, A. Buscher, and S. Asada, "Space-time interface-tracking with topology change (ST-TC)", Computational Mechanics, 54 (2014) 955-971, doi: 10.1007/s00466-013-0935-7.

[56] K. Takizawa, T.E. Tezduyar, and N. Kostov, "Sequentially-coupled spacetime FSI analysis of bio-inspired flapping-wing aerodynamics of an MAV", Computational Mechanics, 54 (2014) 213-233, doi: 10.1007/s00466-0140980-x

[57] K. Takizawa, T.E. Tezduyar, and A. Buscher, "Space-time computational analysis of MAV flapping-wing aerodynamics with wing clapping", Computational Mechanics, 55 (2015) 1131-1141, doi: 10.1007/s00466-014-1095-0.

[58] K. Takizawa, K. Schjodt, A. Puntel, N. Kostov, and T.E. Tezduyar, "Patient-specific computer modeling of blood flow in cerebral arteries with aneurysm and stent", Computational Mechanics, 50 (2012) 675-686, doi: 10.1007/s00466-012-0760-4.

[59] K. Takizawa, K. Schjodt, A. Puntel, N. Kostov, and T.E. Tezduyar, "Patient-specific computational analysis of the influence of a stent on the unsteady flow in cerebral aneurysms", Computational Mechanics, 51 (2013) 1061-1073, doi: 10.1007/s00466-012-0790-y.

[60] K. Takizawa, Y. Bazilevs, T.E. Tezduyar, C.C. Long, A.L. Marsden, and K. Schjodt, "ST and ALE-VMS methods for patient-specific cardiovascular fluid mechanics modeling", Mathematical Models and Methods in Applied Sciences, 24 (2014) 2437-2486, doi: 10.1142/S0218202514500250.

[61] H. Suito, K. Takizawa, V.Q.H. Huynh, D. Sze, and T. Ueda, "FSI analysis of the blood flow and geometrical characteristics in the thoracic aorta", 
Computational Mechanics, 54 (2014) 1035-1045, doi: 10.1007/s00466-0141017-1.

[62] K. Takizawa, T.E. Tezduyar, A. Buscher, and S. Asada, "Space-time fluid mechanics computation of heart valve models", Computational Mechanics, 54 (2014) 973-986, doi: 10.1007/s00466-014-1046-9.

[63] K. Takizawa, D. Montes, M. Fritze, S. McIntyre, J. Boben, and T.E. Tezduyar, "Methods for FSI modeling of spacecraft parachute dynamics and cover separation", Mathematical Models and Methods in Applied Sciences, 23 (2013) 307-338, doi: 10.1142/S0218202513400058

[64] K. Takizawa, D. Montes, S. McIntyre, and T.E. Tezduyar, "Spacetime VMS methods for modeling of incompressible flows at high Reynolds numbers", Mathematical Models and Methods in Applied Sciences, 23 (2013) 223-248, doi: 10.1142/s0218202513400022.

[65] K. Takizawa, T.E. Tezduyar, and T. Kuraishi, "Multiscale ST methods for thermo-fluid analysis of a ground vehicle and its tires", Mathematical Models and Methods in Applied Sciences, 25 (2015) 2227-2255, doi: 10.1142/S0218202515400072.

[66] K. Takizawa, T.E. Tezduyar, T. Kuraishi, S. Tabata, and H. Takagi, "Computational thermo-fluid analysis of a disk brake", Computational Mechanics, 57 (2016) 965-977, doi: 10.1007/s00466-016-1272-4.

[67] K. Takizawa, T.E. Tezduyar, and H. Hattori, "Computational analysis of flow-driven string dynamics in turbomachinery", Computers \& Fluids, published online, DOI: 10.1016/j.compfluid.2016.02.019, March 2016, doi: 10.1016/j.compfluid.2016.02.019

[68] K. Takizawa, T.E. Tezduyar, Y. Otoguro, T. Terahara, T. Kuraishi, and H. Hattori, "Turbocharger flow computations with the space-time isogeometric analysis (ST-IGA)", Computers 8 Fluids, pub- 
lished online, DOI: 10.1016/j.compfluid.2016.02.021, March 2016, doi: 10.1016/j.compfluid.2016.02.021.

[69] K. Takizawa, T.E. Tezduyar, S. Asada, and T. Kuraishi, "Spacetime method for flow computations with slip interfaces and topology changes (ST-SI-TC)", Computers \& Fluids, published online, DOI: 10.1016/j.compfluid.2016.05.006, May 2016, doi: 10.1016/j.compfluid.2016.05.006

[70] K. Takizawa, T.E. Tezduyar, and T. Terahara, "Ram-air parachute structural and fluid mechanics computations with the spacetime isogeometric analysis (ST-IGA)", Computers \& Fluids, published online, DOI: 10.1016/j.compfluid.2016.05.027, May 2016, doi: 10.1016/j.compfluid.2016.05.027

[71] T.E. Tezduyar, K. Takizawa, C. Moorman, S. Wright, and J. Christopher, "Space-time finite element computation of complex fluid-structure interactions", International Journal for Numerical Methods in Fluids, 64 (2010) 1201-1218, doi: 10.1002/fld.2221

[72] D. Kamensky, M.-C. Hsu, D. Schillinger, J.A. Evans, A. Aggarwal, Y. Bazilevs, M.S. Sacks, and T.J.R. Hughes, "An immersogeometric variational framework for fluid-structure interaction: Application to bioprosthetic heart valves", Computer Methods in Applied Mechanics and Engineering, 284 (2015) 1005-1053.

[73] V. Kalro and T.E. Tezduyar, "Parallel finite element computation of 3D incompressible flows on MPPs", in W.G. Habashi, editor, Solution Techniques for Large-Scale CFD Problems, John Wiley \& Sons, 1995.

[74] T. Tezduyar, S. Aliabadi, M. Behr, A. Johnson, V. Kalro, and M. Litke, "Flow simulation and high performance computing", Computational Mechanics, 18 (1996) 397-412, doi: 10.1007/BF00350249. 
[75] M. Behr and T. Tezduyar, "The Shear-Slip Mesh Update Method", Computer Methods in Applied Mechanics and Engineering, 174 (1999) 261-274, doi: 10.1016/S0045-7825(98)00299-0.

[76] Y. Bazilevs, A. Korobenko, X. Deng, and J. Yan, "Novel structural modeling and mesh moving techniques for advanced FSI simulation of wind turbines", International Journal for Numerical Methods in Engineering, 102 (2015) 766-783, doi: 10.1002/nme.4738.

[77] T.E. Tezduyar and D.K. Ganjoo, "Petrov-Galerkin formulations with weighting functions dependent upon spatial and temporal discretization: Applications to transient convection-diffusion problems", Computer Methods in Applied Mechanics and Engineering, 59 (1986) 49-71, doi: 10.1016/0045-7825(86)90023-X.

[78] G.J. Le Beau, S.E. Ray, S.K. Aliabadi, and T.E. Tezduyar, "SUPG finite element computation of compressible flows with the entropy and conservation variables formulations", Computer Methods in Applied Mechanics and Engineering, 104 (1993) 397-422, doi: 10.1016/0045-7825(93)90033-T,

[79] T.E. Tezduyar, "Finite elements in fluids: Stabilized formulations and moving boundaries and interfaces", Computers \&3 Fluids, 36 (2007) 191-206, doi: $10.1016 /$ j.compfluid.2005.02.011.

[80] T.E. Tezduyar and M. Senga, "Stabilization and shock-capturing parameters in SUPG formulation of compressible flows", Computer Methods in Applied Mechanics and Engineering, 195 (2006) 1621-1632, doi: 10.1016/j.cma.2005.05.032.

[81] T.E. Tezduyar and M. Senga, "SUPG finite element computation of inviscid supersonic flows with YZ $\beta$ shock-capturing", Computers \& Fluids, 36 (2007) 147-159, doi: 10.1016/j.compfluid.2005.07.009.

[82] T.E. Tezduyar, M. Senga, and D. Vicker, "Computation of inviscid supersonic flows around cylinders and spheres with the SUPG formulation and 
YZ $\beta$ shock-capturing", Computational Mechanics, 38 (2006) 469-481, doi: 10.1007/s00466-005-0025-6.

[83] T.E. Tezduyar and S. Sathe, "Enhanced-discretization selective stabilization procedure (EDSSP)", Computational Mechanics, 38 (2006) 456-468, doi: $10.1007 / \mathrm{s} 00466-006-0056-7$.

[84] A. Corsini, F. Rispoli, A. Santoriello, and T.E. Tezduyar, "Improved discontinuity-capturing finite element techniques for reaction effects in turbulence computation", Computational Mechanics, 38 (2006) 356-364, doi: 10.1007/s00466-006-0045-x.

[85] F. Rispoli, A. Corsini, and T.E. Tezduyar, "Finite element computation of turbulent flows with the discontinuity-capturing directional dissipation (DCDD)", Computers \& Fluids, 36 (2007) 121-126, doi: 10.1016/j.compfluid.2005.07.004.

[86] T.E. Tezduyar, S. Ramakrishnan, and S. Sathe, "Stabilized formulations for incompressible flows with thermal coupling", International Journal for Numerical Methods in Fluids, 57 (2008) 1189-1209, doi: 10.1002/fld.1743.

[87] F. Rispoli, R. Saavedra, A. Corsini, and T.E. Tezduyar, "Computation of inviscid compressible flows with the V-SGS stabilization and YZ $\beta$ shock-capturing", International Journal for Numerical Methods in Fluids, 54 (2007) 695-706, doi: 10.1002/fld.1447.

[88] Y. Bazilevs, V.M. Calo, T.E. Tezduyar, and T.J.R. Hughes, "YZ $\beta$ discontinuity-capturing for advection-dominated processes with application to arterial drug delivery", International Journal for Numerical Methods in Fluids, 54 (2007) 593-608, doi: 10.1002/fld.1484.

[89] A. Corsini, C. Menichini, F. Rispoli, A. Santoriello, and T.E. Tezduyar, "A multiscale finite element formulation with discontinuity capturing for turbulence models with dominant reactionlike terms", Journal of Applied Mechanics, 76 (2009) 021211, doi: 10.1115/1.3062967. 
[90] F. Rispoli, R. Saavedra, F. Menichini, and T.E. Tezduyar, "Computation of inviscid supersonic flows around cylinders and spheres with the V-SGS stabilization and YZ $\beta$ shock-capturing", Journal of Applied Mechanics, 76 (2009) 021209, doi: 10.1115/1.3057496.

[91] A. Corsini, C. Iossa, F. Rispoli, and T.E. Tezduyar, "A DRD finite element formulation for computing turbulent reacting flows in gas turbine combustors", Computational Mechanics, 46 (2010) 159-167, doi: 10.1007/s00466009-0441-0.

[92] M.-C. Hsu, Y. Bazilevs, V.M. Calo, T.E. Tezduyar, and T.J.R. Hughes, "Improving stability of stabilized and multiscale formulations in flow simulations at small time steps", Computer Methods in Applied Mechanics and Engineering, 199 (2010) 828-840, doi: 10.1016/j.cma.2009.06.019.

[93] A. Corsini, F. Rispoli, and T.E. Tezduyar, "Stabilized finite element computation of NOx emission in aero-engine combustors", International Journal for Numerical Methods in Fluids, 65 (2011) 254-270, doi: 10.1002/fld.2451.

[94] A. Corsini, F. Rispoli, and T.E. Tezduyar, "Computer modeling of waveenergy air turbines with the SUPG/PSPG formulation and discontinuitycapturing technique", Journal of Applied Mechanics, 79 (2012) 010910, doi: $10.1115 / 1.4005060$.

[95] A. Corsini, F. Rispoli, A.G. Sheard, and T.E. Tezduyar, "Computational analysis of noise reduction devices in axial fans with stabilized finite element formulations", Computational Mechanics, 50 (2012) 695-705, doi: 10.1007/s00466-012-0789-4.

[96] P.A. Kler, L.D. Dalcin, R.R. Paz, and T.E. Tezduyar, "SUPG and discontinuity-capturing methods for coupled fluid mechanics and electrochemical transport problems", Computational Mechanics, 51 (2013) 171185, doi: $10.1007 / \mathrm{s} 00466-012-0712-\mathrm{z}$. 
[97] A. Corsini, F. Rispoli, A.G. Sheard, K. Takizawa, T.E. Tezduyar, and P. Venturini, "A variational multiscale method for particle-cloud tracking in turbomachinery flows", Computational Mechanics, 54 (2014) 1191-1202, doi: $10.1007 / \mathrm{s} 00466-014-1050-0$

[98] F. Rispoli, G. Delibra, P. Venturini, A. Corsini, R. Saavedra, and T.E. Tezduyar, "Particle tracking and particle-shock interaction in compressibleflow computations with the V-SGS stabilization and YZ $\beta$ shock-capturing", Computational Mechanics, 55 (2015) 1201-1209, doi: 10.1007/s00466-0151160-3.

[99] Y. Bazilevs and T.J.R. Hughes, "Weak imposition of Dirichlet boundary conditions in fluid mechanics", Computers and Fluids, 36 (2007) 12-26. 


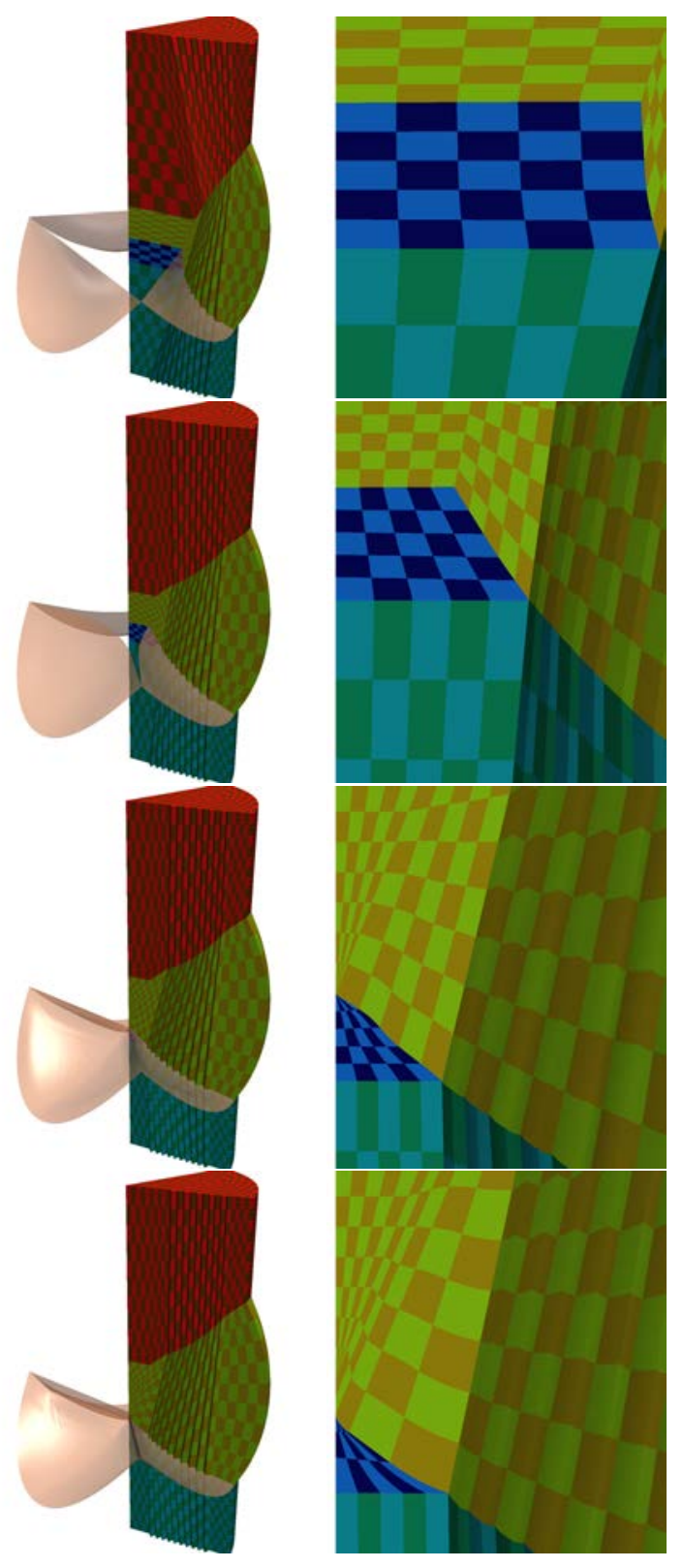

Figure 8: A set of selected NURBS elements, from when the valve is fully open (top) to when it is fully closed (bottom). The corresponding $\theta$ values are $0.0,0.42,0.97$, and 1.0. The right pictures are the zoomed views around the leaflet. 




Figure 9: Inflow velocity (two cycles). 


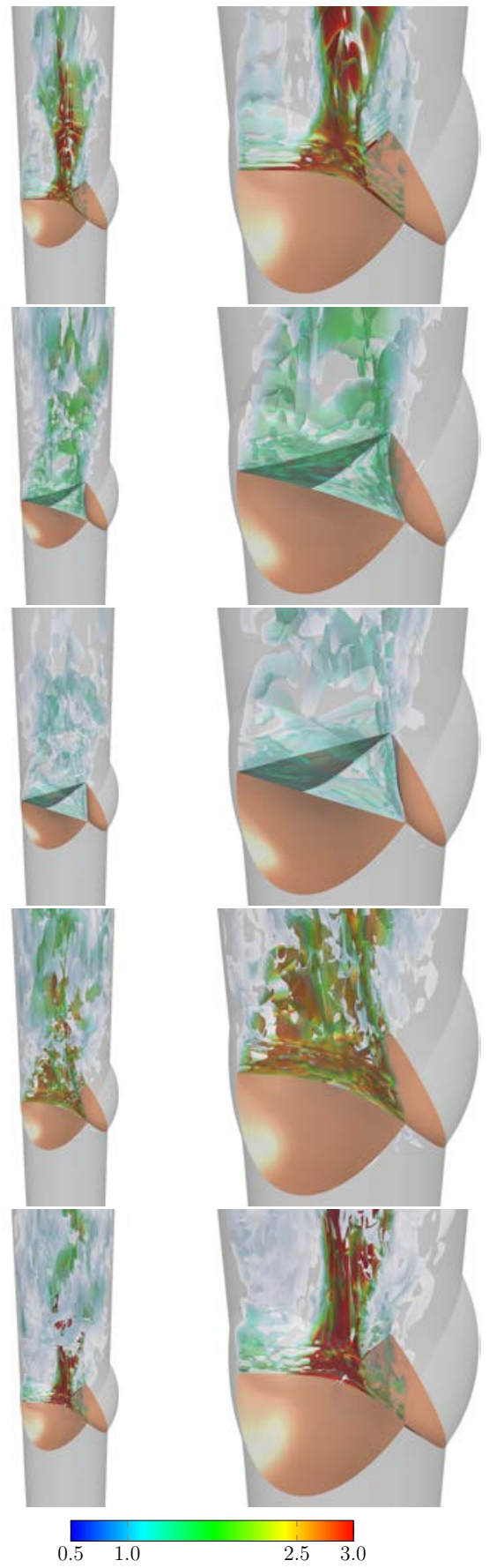

Figure 10: Symmetric leaflet motion. Isosurfaces corresponding to a positive value of the second invariant of the velocity gradient tensor, colored by the velocity magnitude $(\mathrm{m} / \mathrm{s})$. The frames are for $t=0.716,0.804,0.892,0.984$, and $1.028 \mathrm{~s}$. 


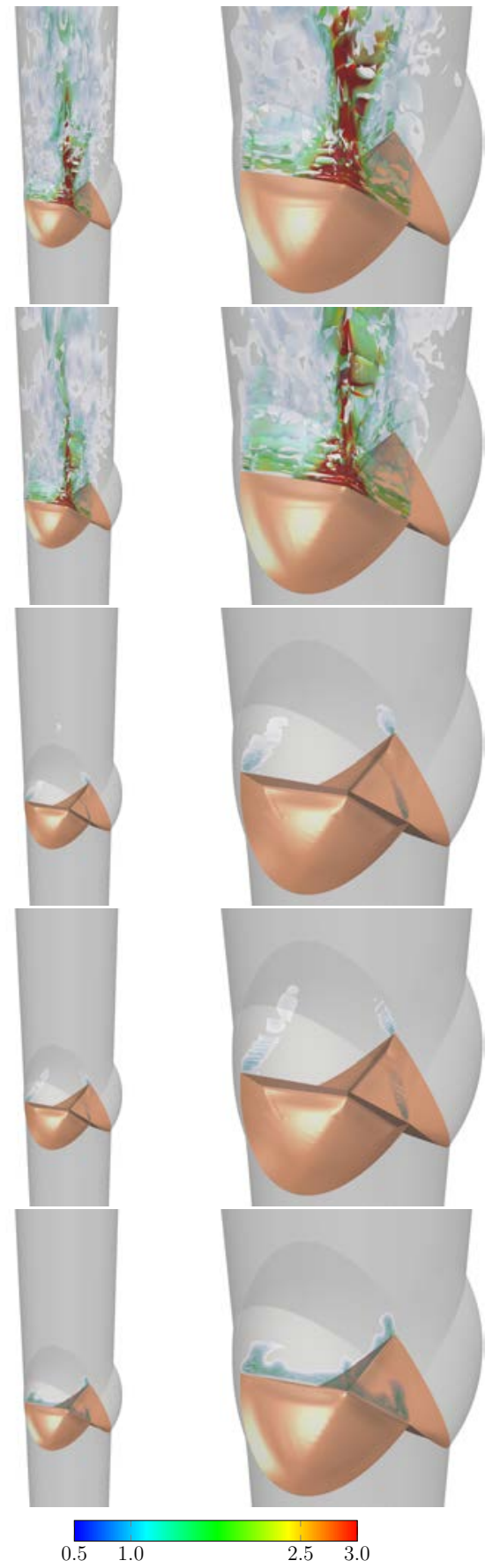

Figure 11: Symmetric leaflet motion. Isosurfaces corresponding to a positive value of the second invariant of the velocity gradient tensor, colored by the velocity magnitude $(\mathrm{m} / \mathrm{s})$. The frames are for $t=1.072,1.080,1.160,1.252$, and $1.340 \mathrm{~s}$. 


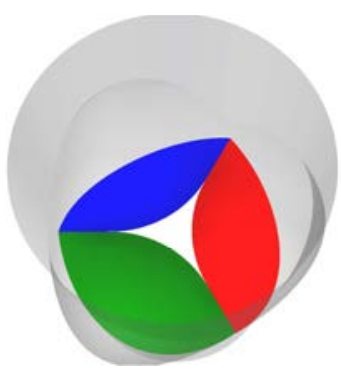

Figure 12: Viewing angle for reporting the WSS. The leaflet identification is same as in Figure 4. 

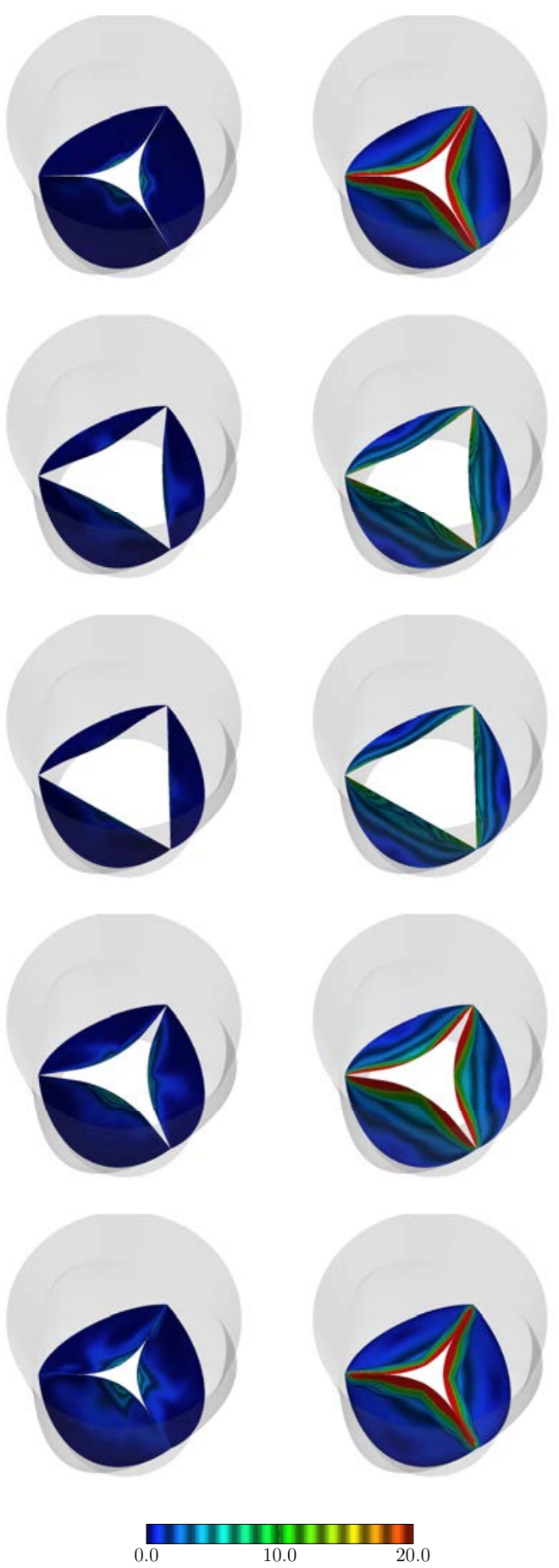

Figure 13: Symmetric leaflet motion. Magnitude of the WSS (Pa). Upper surface (left) and lower surface (right). The frames are for $t=0.716,0.804,0.892,0.984$, and $1.028 \mathrm{~s}$. 

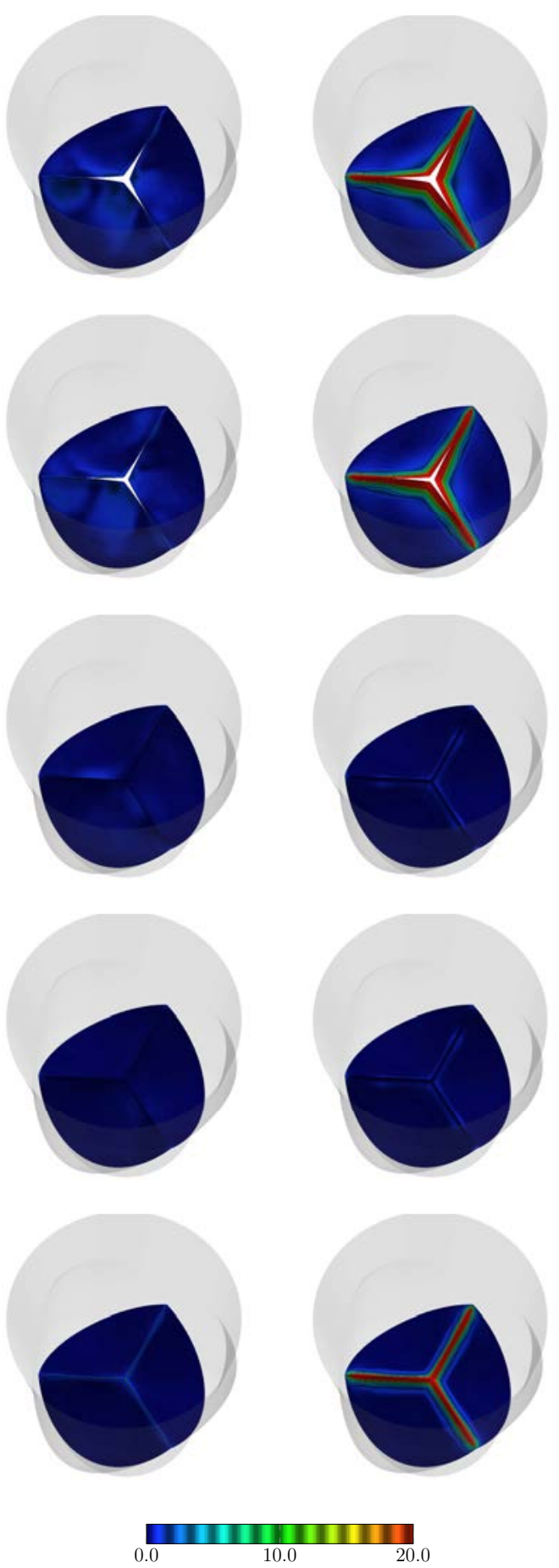

Figure 14: Symmetric leaflet motion. Magnitude of the WSS (Pa). Upper surface (left) and lower surface (right). The frames are for $t=1.072,1.080,1.160,1.252$, and $1.340 \mathrm{~s}$. 


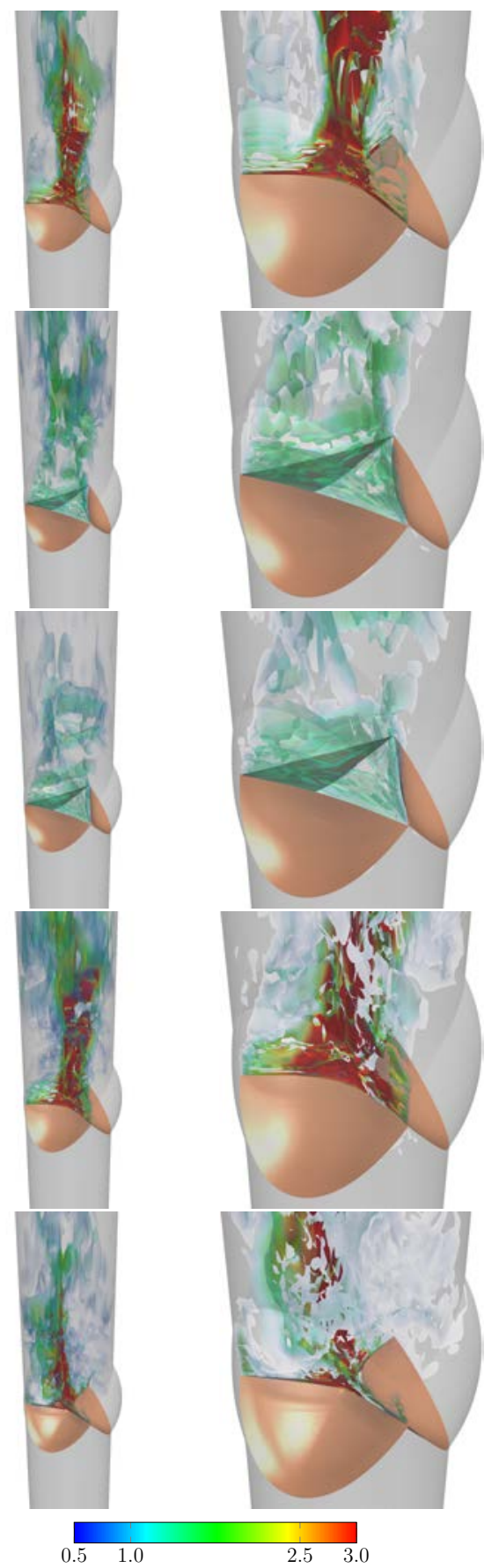

Figure 15: Asymmetric leaflet motion. Isosurfaces corresponding to a positive value of the second invariant of the velocity gradient tensor, colored by the velocity magnitude $(\mathrm{m} / \mathrm{s})$. The frames are for $t=0.716,0.804,0.892,0.984$, and $1.028 \mathrm{~s}$. 


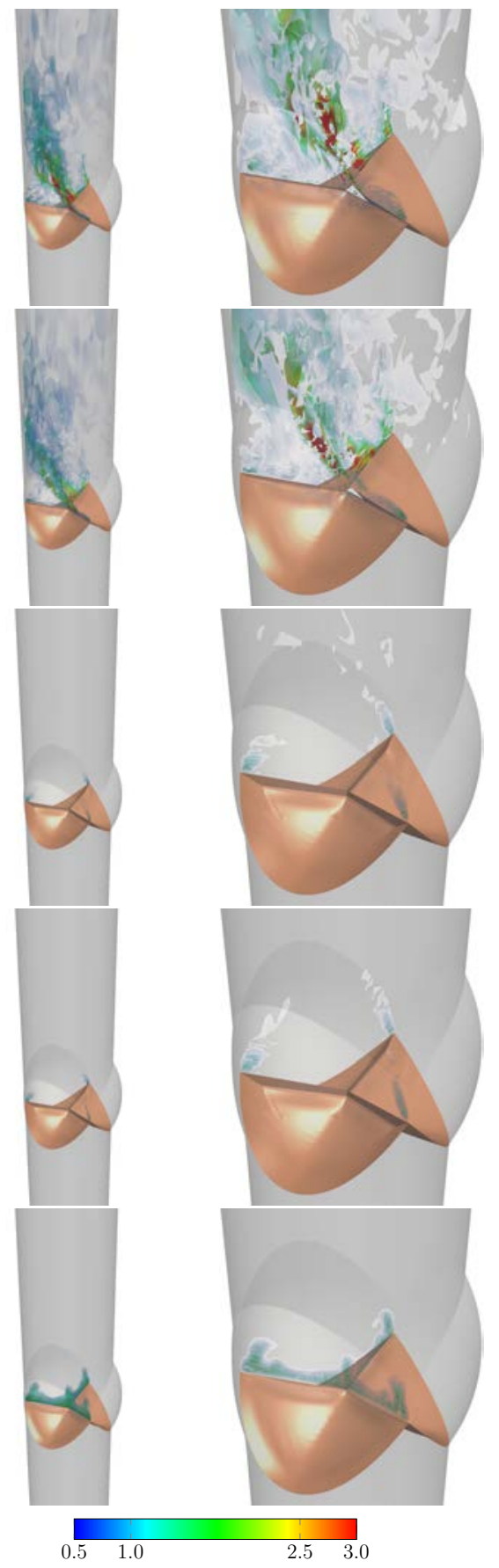

Figure 16: Asymmetric leaflet motion. Isosurfaces corresponding to a positive value of the second invariant of the velocity gradient tensor, colored by the velocity magnitude $(\mathrm{m} / \mathrm{s})$. The frames are for $t=1.072,1.080,1.160,1.252$, and $1.340 \mathrm{~s}$. 

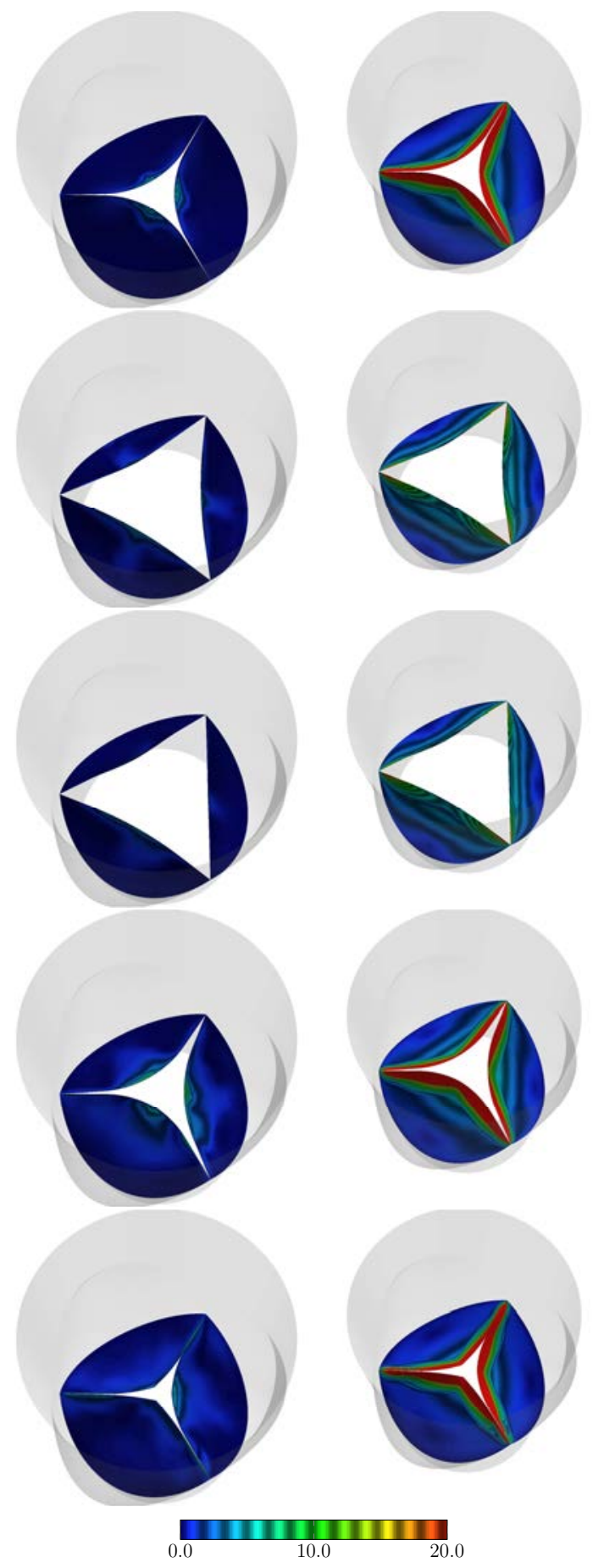

Figure 17: Asymmetric leaflet motion. Magnitude of the WSS (Pa). Upper surface (left) and lower surface (right). The frames are for $t=0.716,0.804,0.892,0.984$, and $1.028 \mathrm{~s}$. 

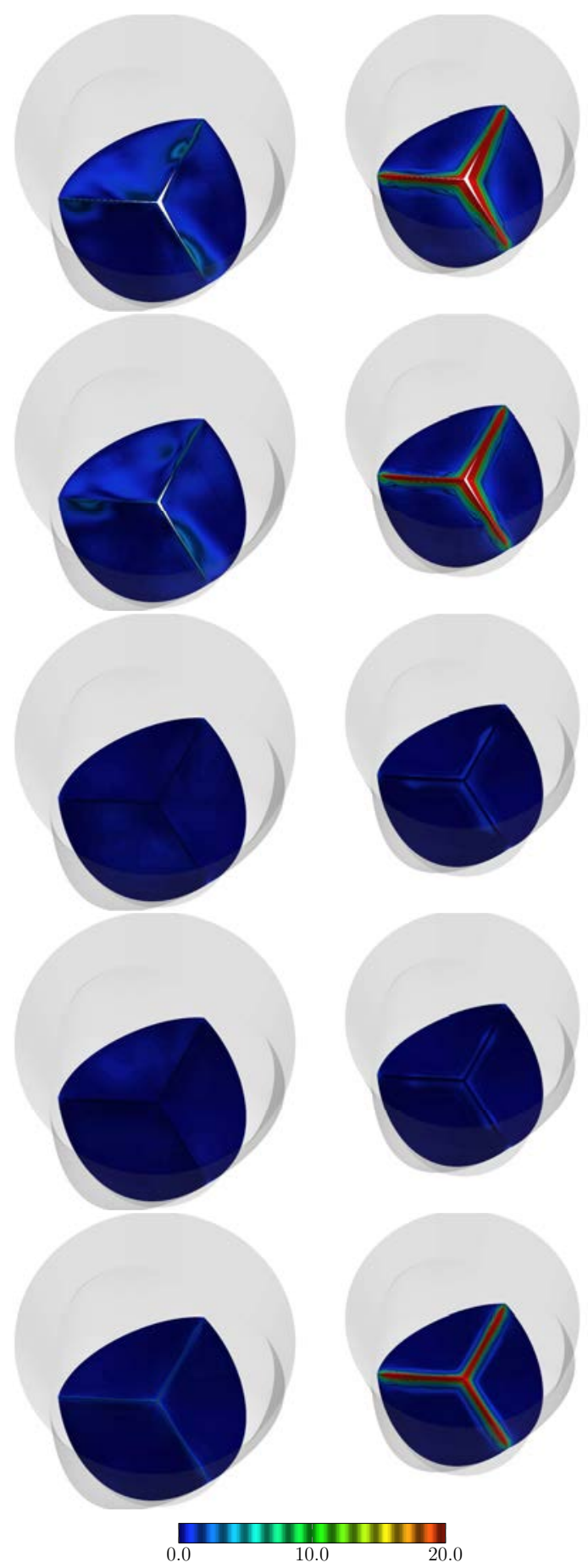

Figure 18: Asymmetric leaflet motion. Magnitude of the WSS (Pa). Upper surface (left) and lower surface (right). The frames are for $t=1.072,1.080,1.160,1.252$, and $1.340 \mathrm{~s}$. 